\title{
Supplementary Index
}

Table 1. Physical Measurements and Analytical Data of Metal(II) Complexes.

\begin{tabular}{|c|c|c|c|c|c|c|c|c|}
\hline \multirow[t]{2}{*}{ No } & \multirow[t]{2}{*}{ Structure } & \multirow{2}{*}{$\begin{array}{l}\text { Yield } \\
(\%)\end{array}$} & \multirow[t]{2}{*}{ MW/ Formula } & \multirow{2}{*}{$\begin{array}{l}\text { M.P } \\
\left({ }^{\circ} \mathrm{C}\right)\end{array}$} & \multicolumn{4}{|c|}{$\begin{array}{c}\text { Elemental Analysis (\%) } \\
\text { Calc (Found) }\end{array}$} \\
\hline & & & & & $\mathrm{C}$ & $\mathrm{H}$ & $\mathrm{N}$ & $\mathrm{M}$ \\
\hline $\mathrm{Co}\left(\mathrm{L}^{1}\right)_{2}$ & {$\left[\mathrm{Co}\left(\mathrm{L}^{1}-\mathrm{H}\right)_{2}\right]$} & 78 & $\begin{array}{c}{[569.69]} \\
\mathrm{C}_{22} \mathrm{H}_{30} \mathrm{~N}_{4} \mathrm{~S}_{4} \mathrm{O}_{2} \mathrm{Co}\end{array}$ & $149-150$ & $\begin{array}{r}46.38 \\
(46.36)\end{array}$ & $\begin{array}{c}5.31 \\
(5.28)\end{array}$ & $\begin{array}{l}9.83 \\
(9.80)\end{array}$ & $\begin{array}{c}10.34 \\
(10.31)\end{array}$ \\
\hline $\mathrm{Ni}\left(\mathrm{L}^{1}\right)_{2}$ & {$\left[\mathrm{Ni}\left(\mathrm{L}^{1}-\mathrm{H}\right)_{2}\right]$} & 81 & $\begin{array}{c}{[569.45]} \\
\mathrm{C}_{22} \mathrm{H}_{30} \mathrm{~N}_{4} \mathrm{~S}_{4} \mathrm{O}_{2} \mathrm{Ni}\end{array}$ & $154-155$ & $\begin{array}{r}46.40 \\
(46.44)\end{array}$ & $\begin{array}{c}5.31 \\
(5.29)\end{array}$ & $\begin{array}{c}9.84 \\
(9.88)\end{array}$ & $\begin{array}{c}10.31 \\
(10.33)\end{array}$ \\
\hline $\mathrm{Cu}\left(\mathrm{L}^{1}\right)_{2}$ & {$\left[\mathrm{Cu}\left(\mathrm{L}^{1}-\mathrm{H}\right)_{2}\right]$} & 82 & $\begin{array}{c}{[574.30]} \\
\mathrm{C}_{22} \mathrm{H}_{30} \mathrm{~N}_{4} \mathrm{~S}_{4} \mathrm{O}_{2} \mathrm{Cu}\end{array}$ & $145-146$ & $\begin{array}{l}46.01 \\
(46.03)\end{array}$ & $\begin{array}{c}5.27 \\
(5.30)\end{array}$ & $\begin{array}{r}9.76 \\
(9.74)\end{array}$ & $\begin{array}{c}11.07 \\
(11.11)\end{array}$ \\
\hline $\mathrm{Zn}\left(\mathrm{L}^{1}\right)_{2}$ & {$\left[\mathrm{Zn}\left(\mathrm{L}^{1}-\mathrm{H}\right)_{2}\right]$} & 76 & $\begin{array}{c}{[576.17]} \\
\mathrm{C}_{22} \mathrm{H}_{30} \mathrm{~N}_{4} \mathrm{~S}_{4} \mathrm{O}_{2} \mathrm{Zn}\end{array}$ & $160-161$ & $\begin{array}{r}45.86 \\
(45.82)\end{array}$ & $\begin{array}{c}5.25 \\
(5.22)\end{array}$ & $\begin{array}{c}9.72 \\
(9.74)\end{array}$ & $\begin{array}{c}11.35 \\
(10.30)\end{array}$ \\
\hline $\mathrm{Co}\left(\mathrm{L}^{2}\right)_{2}$ & {$\left[\mathrm{Co}\left(\mathrm{L}^{2}-\mathrm{H}\right)_{2}\right]$} & 77 & $\begin{array}{c}{[597.74]} \\
\mathrm{C}_{24} \mathrm{H}_{34} \mathrm{~N}_{4} \mathrm{~S}_{4} \mathrm{O}_{2} \mathrm{Co}\end{array}$ & $141-142$ & $\begin{array}{c}48.22 \\
(48.24)\end{array}$ & $\begin{array}{c}5.73 \\
(5.69)\end{array}$ & $\begin{array}{c}9.37 \\
(9.35)\end{array}$ & $\begin{array}{r}9.86 \\
(9.82)\end{array}$ \\
\hline $\mathrm{Ni}\left(\mathrm{L}^{2}\right)_{2}$ & {$\left[\mathrm{Ni}\left(\mathrm{L}^{2}-\mathrm{H}\right)_{2}\right]$} & 80 & $\begin{array}{c}{[597.51]} \\
\mathrm{C}_{24} \mathrm{H}_{34} \mathrm{~N}_{4} \mathrm{~S}_{4} \mathrm{O}_{2} \mathrm{Ni}\end{array}$ & $148-149$ & $\begin{array}{r}48.24 \\
(48.21)\end{array}$ & $\begin{array}{c}5.74 \\
(5.75)\end{array}$ & $\begin{array}{c}9.38 \\
(9.35)\end{array}$ & $\begin{array}{l}9.82 \\
(9.89)\end{array}$ \\
\hline $\mathrm{Cu}\left(\mathrm{L}^{2}\right)_{2}$ & {$\left[\mathrm{Cu}\left(\mathrm{L}^{2}-\mathrm{H}\right)_{2}\right]$} & 79 & $\begin{array}{c}{[602.35]} \\
\mathrm{C}_{24} \mathrm{H}_{34} \mathrm{~N}_{4} \mathrm{~S}_{4} \mathrm{O}_{2} \mathrm{Cu}\end{array}$ & $153-154$ & $\begin{array}{r}47.85 \\
(47.89)\end{array}$ & $\begin{array}{c}5.69 \\
(5.71)\end{array}$ & $\begin{array}{r}9.30 \\
(9.33)\end{array}$ & $\begin{array}{c}10.55 \\
(10.53)\end{array}$ \\
\hline $\mathrm{Zn}\left(\mathrm{L}^{2}\right)_{2}$ & {$\left[\mathrm{Zn}\left(\mathrm{L}^{2}-\mathrm{H}\right)_{2}\right]$} & 78 & $\begin{array}{c}{[604.22]} \\
\mathrm{C}_{24} \mathrm{H}_{34} \mathrm{~N}_{4} \mathrm{~S}_{4} \mathrm{O}_{2} \mathrm{Zn}\end{array}$ & $148-149$ & $\begin{array}{c}47.71 \\
(47.75)\end{array}$ & $\begin{array}{c}5.67 \\
(5.69)\end{array}$ & $\begin{array}{c}9.27 \\
(9.21)\end{array}$ & $\begin{array}{c}10.83 \\
(10.80)\end{array}$ \\
\hline $\mathrm{Co}\left(\mathrm{L}^{3}\right)_{2}$ & {$\left[\mathrm{Co}\left(\mathrm{L}^{3}-\mathrm{H}\right)_{2}\right]$} & 75 & $\begin{array}{c}{[595.35]} \\
\mathrm{C}_{22} \mathrm{H}_{28} \mathrm{Br}_{2} \mathrm{~N}_{4} \mathrm{~S}_{2} \mathrm{O}_{4} \mathrm{Co}\end{array}$ & $138-139$ & $\begin{array}{c}38.00 \\
(38.12)\end{array}$ & $\begin{array}{c}4.06 \\
(4.09)\end{array}$ & $\begin{array}{r}8.06 \\
(8.10)\end{array}$ & $\begin{array}{c}8.48 \\
(8.50)\end{array}$ \\
\hline $\mathrm{Ni}\left(\mathrm{L}^{3}\right)_{2}$ & {$\left[\mathrm{Ni}\left(\mathrm{L}^{3}-\mathrm{H}\right)_{2}\right]$} & 79 & $\begin{array}{c}{[695.11]} \\
\mathrm{C}_{22} \mathrm{H}_{28} \mathrm{Br}_{2} \mathrm{~N}_{4} \mathrm{~S}_{2} \mathrm{O}_{4} \mathrm{Ni}\end{array}$ & $146-147$ & $\begin{array}{c}38.01 \\
(38.05)\end{array}$ & $\begin{array}{c}4.06 \\
(4.07)\end{array}$ & $\begin{array}{r}8.06 \\
(8.02)\end{array}$ & $\begin{array}{c}8.44 \\
(8.49)\end{array}$ \\
\hline $\mathrm{Cu}\left(\mathrm{L}^{3}\right)_{2}$ & {$\left[\mathrm{Cu}\left(\mathrm{L}^{3}-\mathrm{H}\right)_{2}\right]$} & 80 & $\begin{array}{c}{[699.93]} \\
\mathrm{C}_{22} \mathrm{H}_{28} \mathrm{Br}_{2} \mathrm{~N}_{4} \mathrm{~S}_{2} \mathrm{O}_{4} \mathrm{Cu}\end{array}$ & $141-142$ & $\begin{array}{c}37.75 \\
(37.79)\end{array}$ & $\begin{array}{c}4.03 \\
(4.07)\end{array}$ & $\begin{array}{r}8.00 \\
(8.10)\end{array}$ & $\begin{array}{l}9.08 \\
(9.06)\end{array}$ \\
\hline $\mathrm{Zn}\left(\mathrm{L}^{3}\right)_{2}$ & {$\left[\mathrm{Zn}\left(\mathrm{L}^{3}-\mathrm{H}\right)_{2}\right]$} & 75 & $\begin{array}{c}{[701.83]} \\
\mathrm{C}_{22} \mathrm{H}_{28} \mathrm{Br}_{2} \mathrm{~N}_{4} \mathrm{~S}_{2} \mathrm{O}_{4} \mathrm{Zn}\end{array}$ & $150-151$ & $\begin{array}{c}37.65 \\
(37.66)\end{array}$ & $\begin{array}{c}4.02 \\
(4.05)\end{array}$ & $\begin{array}{c}7.98 \\
(7.95)\end{array}$ & $\begin{array}{c}9.32 \\
(9.38)\end{array}$ \\
\hline
\end{tabular}


Table 3. Crystal Data and Details Structure Determinations of Ligand $\left(\mathbf{L}^{\mathbf{1}}\right)$ and their $\mathbf{C u}\left(\mathbf{L}^{\mathbf{1}}\right)_{2}$ Complex.

\begin{tabular}{|c|c|c|}
\hline Compound & $\left(\mathbf{L}^{1}\right)$ & $\mathrm{Cu}\left(\mathbf{L}^{1}\right)_{2}$ \\
\hline Empirical formula & $\mathrm{C}_{11} \mathrm{H}_{16} \mathrm{~N}_{2} \mathrm{OS}_{2}$ & $\mathrm{C}_{22} \mathrm{H}_{30} \mathrm{CuN}_{4} \mathrm{O}_{2} \mathrm{~S}_{4}$ \\
\hline Formula weight & 256.38 & 574.28 \\
\hline Temperature & $100 \mathrm{~K}$ & $100 \mathrm{~K}$ \\
\hline Colour & Colourless & Green \\
\hline Crystal system, space group & Monoclinic, $P 2_{1} / c$ & Monoclinic, $P 2_{1} / c$ \\
\hline A & $12.1709(18) \AA$ & $11.178(3) \AA$ \\
\hline B & $7.7705(12) \AA$ & $15.942(4) \AA$ \\
\hline $\mathrm{C}$ & $14.510(2) \AA$ & $15.013(4) \AA$ \\
\hline B & $106.062(2)^{\circ}$ & $101.385(5)^{\circ}$ \\
\hline V & $1318.7(3) \AA^{3}$ & $2622.7(11) \AA^{3}$ \\
\hline Z, Calculated density & $4,1.291 \mathrm{Mg} \mathrm{m}^{-3}$ & $4,1.454 \mathrm{Mg} \mathrm{m}^{-3}$ \\
\hline Absorption coefficient $(\mu)$ & $0.39 \mathrm{~mm}^{-1}$ & $1.18 \mathrm{~mm}^{-1}$ \\
\hline Crystal size & $0.50 \times 0.40 \times 0.40 \mathrm{~mm}$ & $0.50 \times 0.50 \times 0.40 \mathrm{~mm}$ \\
\hline $\mathrm{F}(000)$ & 544 & 1196 \\
\hline Theta range for data collection $(\theta)$ & $3.7-26.3^{\circ}$ & $2.5-23.2^{\circ}$ \\
\hline Parameters & 903 & 1020 \\
\hline$\theta_{\max }, \theta_{\min }$ & $26.4^{\circ}, 2.9^{\circ}$ & $26.3^{\circ}, 1.9^{\circ}$ \\
\hline$R_{\text {int }}$ & 0.063 & 0.053 \\
\hline Measured reflections & 10074 & 14594 \\
\hline Unique reflections & 2688 & 5301 \\
\hline Observed reflections $I>2 \sigma(I)$ & 2360 & 3222 \\
\hline$R\left[F^{2}>2 \sigma\left(F^{2}\right)\right]$ & 0.035 & 0.058 \\
\hline$w R\left(F^{2}\right)$ & 0.104 & 0.139 \\
\hline$(\Delta / \sigma)_{\max }$ & $<0.001$ & $<0.001$ \\
\hline$\Delta \rho_{\max }, \Delta \rho_{\min }$ & 0.45 e $\AA^{-3},-0.30$ e $\AA^{-3}$ & 0.64 e $\AA^{-3},-0.49$ e $\AA^{-3}$ \\
\hline CCDC number & 805690 & 774651 \\
\hline Cif file & $\mathrm{s} 3351 \mathrm{~m}$ & $\mathrm{~S} 3359 \mathrm{~m}$ \\
\hline
\end{tabular}


Table 4. Selected Bond Lengths ( $\mathrm{A})$ of Ligand $\left(\mathbf{L}^{\mathbf{1}}\right)$.

\begin{tabular}{llll}
\hline Atoms & Bond Length $(\AA)$ & Atoms & Bond Length $(\AA)$ \\
\hline S2-C6 & $1.6792(16)$ & $\mathrm{S} 1-\mathrm{C} 4$ & $1.700(2)$ \\
$\mathrm{O} 1-\mathrm{C} 5$ & $1.221(2)$ & $\mathrm{S} 1-\mathrm{C} 1$ & $1.7199(16)$ \\
$\mathrm{N} 1-\mathrm{C} 5$ & $1.374(2)$ & $\mathrm{C} 1-\mathrm{C} 2$ & $1.413(2)$ \\
$\mathrm{N} 1-\mathrm{C} 6$ & $1.417(2)$ & $\mathrm{C} 1-\mathrm{C} 5$ & $1.469(2)$ \\
$\mathrm{N} 2-\mathrm{C} 6$ & $1.323(2)$ & $\mathrm{C} 2-\mathrm{C} 3$ & $1.411(2)$ \\
$\mathrm{N} 1-\mathrm{C} 7$ & $1.475(2)$ & $\mathrm{C} 3-\mathrm{C} 4$ & $1.357(3)$ \\
$\mathrm{N} 1-\mathrm{C} 9$ & $1.493(2)$ & $\mathrm{C} 2-\mathrm{H} 2$ & 0.9500 \\
$\mathrm{C} 7-\mathrm{C} 8$ & $1.519(2)$ & $\mathrm{C} 3-\mathrm{H} 3$ & 0.9500 \\
$\mathrm{C} 9-\mathrm{C} 11$ & $1.525(3)$ & $\mathrm{C} 4-\mathrm{H} 4$ & 0.9500 \\
\hline
\end{tabular}

Table 5. Selected Bond Angles (deg) of Ligand ( $\left.\mathbf{L}^{\mathbf{1}}\right)$.

\begin{tabular}{llll}
\hline Atoms & $\begin{array}{l}\text { Bond Angle } \\
\text { degree }\end{array}$ & Atoms & $\begin{array}{l}\text { Bond Angle } \\
\text { (degree }\end{array}$ \\
\hline C11-C9-C10 & $113.26(16)$ & $\mathrm{C} 3-\mathrm{C} 4-\mathrm{S} 1$ & $112.51(13)$ \\
$\mathrm{C} 5-\mathrm{N} 1-\mathrm{C} 6$ & $123.52(14)$ & $\mathrm{C} 4-\mathrm{S} 1-\mathrm{C} 1$ & $91.98(9)$ \\
$\mathrm{C} 7-\mathrm{N} 2-\mathrm{C} 9$ & $116.62(13)$ & $\mathrm{C} 2-\mathrm{C} 1-\mathrm{C} 5$ & $130.21(14)$ \\
$\mathrm{C} 3-\mathrm{C} 2-\mathrm{C} 1$ & $110.91(14)$ & $\mathrm{C} 5-\mathrm{C} 1-\mathrm{S} 1$ & $118.78(12)$ \\
$\mathrm{N} 2-\mathrm{C} 7-\mathrm{C} 8$ & $112.72(14)$ & $\mathrm{C} 2-\mathrm{C} 1-\mathrm{S} 1$ & $110.99(12)$ \\
$\mathrm{O} 1-\mathrm{C} 5-\mathrm{N} 1$ & $122.48(15)$ & $\mathrm{C} 4-\mathrm{C} 3-\mathrm{C} 2$ & $113.58(16)$ \\
$\mathrm{N} 1-\mathrm{C} 5-\mathrm{C} 1$ & $114.71(14)$ & $\mathrm{N} 2-\mathrm{C} 6-\mathrm{N} 1$ & $116.87(14)$ \\
$\mathrm{N} 2-\mathrm{C} 6-\mathrm{S} 2$ & $125.63(12)$ & $\mathrm{N} 1-\mathrm{C} 6-\mathrm{S} 2$ & $117.48(12)$ \\
$\mathrm{N} 2-\mathrm{C} 9-\mathrm{C} 11$ & $111.19(15)$ & $\mathrm{C} 6-\mathrm{N} 2-\mathrm{C} 9$ & $123.53(13)$ \\
$\mathrm{C} 6-\mathrm{N} 2-\mathrm{C} 7$ & $119.31(14)$ & $\mathrm{N} 2-\mathrm{C} 9-\mathrm{C} 10$ & $109.39(14)$ \\
$\mathrm{C} 6-\mathrm{N} 2-\mathrm{C} 7-\mathrm{C} 8$ & $81.11(19)$ & $\mathrm{C} 6-\mathrm{N} 2-\mathrm{C} 9-\mathrm{C} 11$ & $130.10(17)$ \\
$\mathrm{S} 1-\mathrm{C} 1-\mathrm{C} 5-\mathrm{N} 1$ & $-173.48(11)$ & $\mathrm{C} 7-\mathrm{N} 2-\mathrm{C} 6-\mathrm{N} 1$ & $-178.58(14)$ \\
$\mathrm{C} 7-\mathrm{N} 2-\mathrm{C} 6-\mathrm{S} 2$ & $3.1(2)$ & $\mathrm{C} 9-\mathrm{N} 2-\mathrm{C} 6-\mathrm{N} 1$ & $10.2(2)$ \\
$\mathrm{C} 9-\mathrm{N} 2-\mathrm{C} 6-\mathrm{S} 2$ & $-168.13(13)$ & $\mathrm{C} 5-\mathrm{N} 1-\mathrm{C} 6-\mathrm{N} 2$ & $69.0(2)$ \\
$\mathrm{C} 5-\mathrm{N} 1-\mathrm{C} 6-\mathrm{S} 2$ & $-112.54(15)$ & $\mathrm{C} 6-\mathrm{N} 2-\mathrm{C} 9-\mathrm{C} 10$ & $104.07(18)$
\end{tabular}




$\begin{array}{llll}\mathrm{C} 6-\mathrm{N} 1-\mathrm{C} 5-\mathrm{O} 1 & -9.4(2) & \mathrm{C} 7-\mathrm{N} 2-\mathrm{C} 9-\mathrm{C} 10 & -67.40(18) \\ \mathrm{C} 6-\mathrm{N} 1-\mathrm{C} 5-\mathrm{C} 1 & 171.47(14) & \mathrm{C} 7-\mathrm{N} 2-\mathrm{C} 9-\mathrm{C} 11 & -130.10(17) \\ \mathrm{C} 2-\mathrm{C} 1-\mathrm{C} 5-\mathrm{O} 1 & -174.45(15) & \mathrm{C} 9-\mathrm{N} 2-\mathrm{C} 7-\mathrm{C} 8 & -107.05(17) \\ \mathrm{S} 1-\mathrm{C} 1-\mathrm{C} 5-\mathrm{O} 1 & 7.4(2) & \mathrm{C} 2-\mathrm{C} 1-\mathrm{C} 5-\mathrm{N} 1 & 4.6(2) \\ \mathrm{C} 2-\mathrm{S} 1-\mathrm{C} 1-\mathrm{C} 2 & 0.91(12) & \mathrm{C} 4-\mathrm{S} 1-\mathrm{C} 1-\mathrm{C} 5 & 179.36(13) \\ \mathrm{C} 1-\mathrm{C} 2-\mathrm{C} 3-\mathrm{C} 4 & 1.7(2) & \mathrm{S} 1-\mathrm{C} 1-\mathrm{C} 2-\mathrm{C} 3 & -1.62(16) \\ \mathrm{C} 1-\mathrm{S} 1-\mathrm{C} 4-\mathrm{C} 3 & 0.06(14) & \mathrm{C} 2-\mathrm{C} 3-\mathrm{C} 4-\mathrm{S} 1 & -1.04(19)\end{array}$

Table 6. Hydrogen Bond Geometry $\left(\AA^{\circ}\right)$ of Ligand $\left(\mathbf{L}^{\mathbf{1}}\right)$.

\begin{tabular}{lllll}
\hline $\mathrm{D}-\mathrm{H}^{\cdots}{ }^{\prime} \mathrm{A}$ & $D-\mathrm{H}$ & $\mathrm{H} \ldots A$ & $\mathrm{D} \ldots A$ & $\mathrm{D}-\mathrm{H} \ldots A$ \\
\hline $\mathrm{N} 1-\mathrm{H} 1 \mathrm{~N}^{\cdots} \mathrm{S} 2^{\mathrm{i}}$ & $0.76(2)$ & $2.73(2)$ & $3.3471(15)$ & $140(19)$ \\
\hline
\end{tabular}

Symmetry code: (i) $-x,-y+2,-z+1$.

Table 7. Selected Bond Lengths $(\AA)$ of $\mathrm{Cu}\left(\mathrm{L}^{1}\right)_{2}$ Complex.

\begin{tabular}{llll}
\hline Atoms & Bond Length $(\AA)$ & Atoms & Bond Length $(\AA)$ \\
\hline $\mathrm{Cu} 1-\mathrm{O} 1$ & $1.941(3)$ & $\mathrm{N} 4-\mathrm{C} 17$ & $1.344(6)$ \\
$\mathrm{Cu} 1-\mathrm{O} 2$ & $1.931(3)$ & $\mathrm{N} 4-\mathrm{C} 18$ & $1.508(7)$ \\
$\mathrm{Cu} 1-\mathrm{S} 1$ & $2.2398(14)$ & $\mathrm{N} 4-\mathrm{C} 21$ & $1.722(14)$ \\
$\mathrm{Cu} 1-\mathrm{S} 3$ & $2.2433(12)$ & $\mathrm{N} 4-\mathrm{C} 18 \mathrm{~B}$ & $1.320(15)$ \\
$\mathrm{O} 1-\mathrm{C} 5$ & $1.273(5)$ & $\mathrm{S} 2-\mathrm{C} 1$ & $1.718(4)$ \\
$\mathrm{O} 2-\mathrm{C} 16$ & $1.267(5)$ & $\mathrm{S} 2-\mathrm{C} 4$ & $1.693(6)$ \\
$\mathrm{S} 1-\mathrm{C} 6$ & $1.746(4)$ & $\mathrm{S} 4-\mathrm{C} 12$ & $1.682(5)$ \\
$\mathrm{S} 3-\mathrm{C} 17$ & $1.716(5)$ & $\mathrm{S} 4-\mathrm{C} 15$ & $1.619(6)$ \\
$\mathrm{N} 1-\mathrm{C} 5$ & $1.310(6)$ & $\mathrm{S} 4-\mathrm{C} 12 \mathrm{~B}$ & $1.637(10)$ \\
$\mathrm{N} 1-\mathrm{C} 6$ & $1.333(6)$ & $\mathrm{C} 1-\mathrm{C} 2$ & $1.384(7)$ \\
$\mathrm{N} 3-\mathrm{C} 16$ & $1.325(6)$ & $\mathrm{C} 1-\mathrm{C} 5$ & $1.495(6)$ \\
$\mathrm{N} 3-\mathrm{C} 17$ & $1.337(6)$ & $\mathrm{C} 7-\mathrm{C} 8$ & $1.507(7)$ \\
$\mathrm{N} 2-\mathrm{C} 10$ & $1.461(6)$ & $\mathrm{C} 7-\mathrm{C} 9$ & $1.543(8)$ \\
$\mathrm{N} 2-\mathrm{C} 6$ & $1.336(5)$ & $\mathrm{N} 2-\mathrm{C} 7$ & $1.503(5)$ \\
\hline
\end{tabular}


Table 8. Selected Bond Angles (deg) of $\mathrm{Cu}\left(\mathrm{L}^{1}\right)_{2}$ Complex.

\begin{tabular}{|c|c|c|c|}
\hline Atoms & $\begin{array}{l}\text { Bond Angle } \\
\text { (degree) }\end{array}$ & Atoms & $\begin{array}{l}\text { Bond Angle } \\
\text { (degree) }\end{array}$ \\
\hline $\mathrm{O} 1-\mathrm{Cu} 1-\mathrm{O} 2$ & $86.24(13)$ & $\mathrm{N} 1-\mathrm{C} 5-\mathrm{C} 1$ & $114.2(4)$ \\
\hline $\mathrm{O} 1-\mathrm{Cu} 1-\mathrm{S} 1$ & $92.84(10)$ & $\mathrm{O} 1-\mathrm{C} 5-\mathrm{C} 1$ & $113.7(4)$ \\
\hline $\mathrm{O} 2-\mathrm{Cu} 1-\mathrm{S} 3$ & $93.64(10)$ & $\mathrm{N} 3-\mathrm{C} 16-\mathrm{C} 12$ & $114.6(4)$ \\
\hline $\mathrm{S} 1-\mathrm{Cu} 1-\mathrm{S} 3$ & $86.84(5)$ & $\mathrm{N} 1-\mathrm{C} 6-\mathrm{N} 2$ & $116.6(4)$ \\
\hline $\mathrm{O} 1-\mathrm{Cu} 1-\mathrm{S} 3$ & $175.18(10)$ & $\mathrm{N} 3-\mathrm{C} 17-\mathrm{N} 4$ & $115.3(4)$ \\
\hline $\mathrm{O} 2-\mathrm{Cu} 1-\mathrm{S} 1$ & $174.71(11)$ & $\mathrm{S} 3-\mathrm{C} 17-\mathrm{N} 4$ & $116.1(4)$ \\
\hline $\mathrm{Cu} 1-\mathrm{O} 1-\mathrm{C} 5$ & $131.0(3)$ & $\mathrm{O} 2-\mathrm{C} 16-\mathrm{C} 12$ & $114.4(4)$ \\
\hline $\mathrm{Cu} 1-\mathrm{O} 2-\mathrm{C} 16$ & $131.7(3)$ & $\mathrm{S} 1-\mathrm{C} 6-\mathrm{N} 2$ & $116.1(4)$ \\
\hline $\mathrm{Cu} 1-\mathrm{S} 1-\mathrm{C} 6$ & $108.83(18)$ & $\mathrm{N} 2-\mathrm{C} 7-\mathrm{C} 8$ & 109.3(4) \\
\hline $\mathrm{Cu} 1-\mathrm{S} 3-\mathrm{C} 17$ & $108.89(19)$ & $\mathrm{C} 1-\mathrm{C} 2-\mathrm{C} 3$ & $108.8(5)$ \\
\hline $\mathrm{O} 1-\mathrm{C} 5-\mathrm{N} 1$ & $132.1(4)$ & $\mathrm{C} 13-\mathrm{C} 14-\mathrm{C} 15$ & $109(8)$ \\
\hline $\mathrm{O} 2-\mathrm{C} 16-\mathrm{N} 3$ & $131.0(5)$ & $\mathrm{C} 2-\mathrm{C} 3-\mathrm{C} 4$ & $113.0(5)$ \\
\hline $\mathrm{N} 1-\mathrm{C} 6-\mathrm{S} 1$ & $127.3(3)$ & $\mathrm{C} 12-\mathrm{C} 13-\mathrm{C} 14$ & $110.7(10)$ \\
\hline $\mathrm{N} 3-\mathrm{C} 17-\mathrm{S} 3$ & $128.6(3)$ & $\mathrm{C} 16-\mathrm{N} 3-\mathrm{C} 17$ & $125.8(4)$ \\
\hline $\mathrm{C} 6-\mathrm{N} 1-\mathrm{C} 5$ & $125.7(4)$ & $\mathrm{C} 17-\mathrm{N} 4-\mathrm{C} 18$ & $122.4(5)$ \\
\hline $\mathrm{O} 1-\mathrm{Cu} 1-\mathrm{S} 3-\mathrm{C} 17$ & $-82.9(12)$ & $\mathrm{O} 1-\mathrm{Cu} 1-\mathrm{S} 1-\mathrm{C} 6$ & $-14.02(17)$ \\
\hline $\mathrm{Cu} 1-\mathrm{O} 1-\mathrm{C} 5-\mathrm{N} 1$ & $-1.4(7)$ & $\mathrm{O} 2-\mathrm{Cu} 1-\mathrm{S} 3-\mathrm{C} 17$ & $5.52(18)$ \\
\hline $\mathrm{Cu} 1-\mathrm{O} 1-\mathrm{C} 5-\mathrm{C} 1$ & $176.5(3)$ & $\mathrm{Cu} 1-\mathrm{S} 1-\mathrm{C} 6-\mathrm{N} 2$ & $-167.4(3)$ \\
\hline $\mathrm{Cu} 1-\mathrm{S} 1-\mathrm{C} 6-\mathrm{N} 1$ & $12.9(4)$ & $\mathrm{Cu} 1-\mathrm{O} 2-\mathrm{C} 16-\mathrm{C} 12$ & $-179.6(3)$ \\
\hline $\mathrm{Cu} 1-\mathrm{O} 2-\mathrm{C} 16-\mathrm{N} 3$ & $1.2(7)$ & $\mathrm{C} 13-\mathrm{C} 12-\mathrm{C} 16-\mathrm{N} 3$ & $172.0(11)$ \\
\hline $\mathrm{Cu} 1-\mathrm{S} 3-\mathrm{C} 17-\mathrm{N} 4$ & $174.3(3)$ & $\mathrm{N} 1-\mathrm{C} 5-\mathrm{C} 1-\mathrm{S} 2$ & $0.3(5)$ \\
\hline $\mathrm{O} 1-\mathrm{C} 5-\mathrm{C} 1-\mathrm{S} 2$ & $-178.0(3)$ & $\mathrm{C} 10-\mathrm{N} 2-\mathrm{C} 7-\mathrm{C} 9$ & $32.2(6)$ \\
\hline $\mathrm{C} 6-\mathrm{N} 2-\mathrm{C} 10-\mathrm{C} 11$ & $88.9(5)$ & $\mathrm{O} 1-\mathrm{C} 5-\mathrm{N} 1-\mathrm{C} 6$ & $-7.2(7)$ \\
\hline
\end{tabular}

